\title{
Tracing the emergence of multidrug-resistant Acinetobacter baumannii in a Taiwanese hospital by evaluating the presence of integron gene int/1
}

\author{
Chang-Hua Chen ${ }^{1,2,3}$ and Chieh-Chen Huang ${ }^{4 *}$
}

\begin{abstract}
Background: In Changhua County, Taiwan, the number of clinical Acinetobacter baumannii isolates has risen since 2002, and multidrug-resistant Acinetobacter baumannii (MDRAB) has spread rapidly throughout Taiwan. In this study, to reveal the mechanism involved with the rapid dissemination of MDRAB emergence, the utility of the class 1 integron, int/1 integrase gene, as an MDRAB-associated biomarker was examined. A cross-sectional, clinical epidemiological study was performed at Changhua Christian Hospital between January $1^{\text {st, }} 2001$ and December 31 $1^{\text {st }}, 2004$. Besides the existence of int/1 gene was examined, the pulse-field gel electrophoresis (PFGE) was also performed to determine the epidemiological characteristics of the isolates.

Findings: The overall hospital infection rate was 5-6\%, while the infection rate of the intensive care unit (ICU) fluctuated. No positive correlation was observed between MDRAB isolates and the presence of int/1 $(r=0.168$, $P=0.254$ ). Additionally, no positive correlation was observed between the infection rate in the ICU and the presence of int/1 ( $r=-0.107, P=0.468)$ or between the hospital infection rate and the presence of intl1 $(r=-0.189, P=0.199)$. However, two predominant clones among the MDRAB isolates were identified by PFGE.

Conclusions: Although the presence of the int/1 gene does not seem suitable for tracing MDRAB emergence in Changhua County, two predominant clones were identified by PFGE, and subsequent studies to identify whether these clones were responsible for original nosocomial infection are needed.
\end{abstract}

Keywords: Acinetobacter baumannii, Int/1 gene, Class 1 integron

\section{Findings \\ Introduction}

Multidrug-resistant Acinetobacter baumannii (MDRAB) is globally recognized as an important cause of nosocomial infections during this century [1]. Both environmental adaptation and rapid acquisition of resistance genes have contributed to the ability of Acinetobacter baumannii (AB) to be such a prosperous pathogen [2]. These resistance genes are primarily spread through horizontal gene transfer (HGT) of plasmids, transposons, or integrons that carry gene clusters that provide resistance to several antibiotic families [3]. Resistance islands (RIs) are important elements for acquiring foreign genes by HGT [4]. The

\footnotetext{
* Correspondence: cchuang@dragon.nchu.edu.tw

${ }^{4}$ College of Life Science, National Chung Hsing University, 250 Kuo-Kuang Road, Taichung 402, Taiwan

Full list of author information is available at the end of the article
}

RIs containing the integrons have been reported in many MDRAB strains [5-8]. Within Changhua County (CC), Taiwan, the numbers of clinical $A B$ isolates have risen since 2002, and the first MDRAB was reported in Taiwan that same year [9]. Since then, MDRAB has rapidly spread through Taiwan and been an emerging problem. Though early detection is critical for isolation and targeted therapy, no well-known biomarker for detecting those mobile characteristics exists because of the structural diversity of RIs of $\mathrm{AB}$. On the other hand, the class 1 integroncarrying MDRAB clones were reported to be widespread in Taiwan [10], while Koeleman showed that amplification of the IntI gene may act as a rapid and simple technique for the routine screening and identification of clinical A. baumannii isolates with epidemic potential [11]. Another study showed that integron detection could be a useful tool for studying molecular epidemiology in hospital environments, facilitating the quick detection of 
possible cross-infection cases [12], especially in critical wards such as the intensive care unit (ICU). To reveal the mechanism involved with the rapid dissemination of MDRAB emergence, the presence of the intI1 integrase gene in class 1 integrons was assessed as an MDRAB-associated biomarker in this study.

\section{Methods}

\section{Clinical investigations}

The Changhua Christian Hospital (CCH) is an 1800-bed tertiary teaching hospital located at CC, Central Taiwan. A cross-sectional, epidemiological study of MDRAB isolates was conducted at $\mathrm{CCH}$ from January $1^{\text {st }}, 2001$ to December $31^{\text {st }}, 2004$. Infectious disease specialists reviewed all patient medical records. Because and our findings disclosed MDRAB, which were resistant to many broad-spectrum antibiotics (including ciprofloxacin, piperacillin-tazobactam, ceftazidime, cefepime, imipenem-cilastatin, amikacin) since 2001-2002 and we reviewed literature (Additional file 1), the critical period for the appearance and spread of MDRAB was estimated to be 2001-2002. We choose the study year is 2001-2002 with the hypothesis of study year is the beginning of the increment of resistant A. baumannii infection. Concerning the observation year, we selected the 2003-2004 for comparison. If the hypothesis is correct, the presence of the intI1 integrase gene in class 1 integrons will increase between the study year and the observation year. The Standard Centers for Disease Control and Prevention National Nosocomial Infections Surveillance definitions for nosocomial infections were used [13]. The hospital infection rate and the ICU infection rate were calculated. We also performed retrospective surveillance for nosocomial infections in the ICUs.

\section{Microbiological investigations}

All AB isolates were routinely examined at Medical Laboratory Department of $\mathrm{CCH}$ and stored in the Bacterial Bank of $\mathrm{CCH}$ from January $1^{\text {st }}, 2001$ to December $31^{\text {st }}$, 2004. Phenotypic methods were used to identify $A$. baumannii isolates using a Vitek-2 System (BioMerieux, Marcy l'Etoile, France). Additionally, antimicrobial susceptibility was determined using an automated Vitek 2 (bioMe'rieux, Marcy l'Étoile, France) according to the recommendations of Clinical and Laboratory Standards Institute [14]. In this study, MDRAB was defined as resistance to at least two specific representatives of at least two classes of antibiotics among those antibiotic categories, including aminoglycosides, anti-pseudomonal penicillins, carbapenems, third or fourth generation cephalosporins and quinolones [15].

\section{Molecular detection of Int/1 integrase gene}

As previously described, the Koeleman's method was used to amplify the targeted intI1 gene [11]. We used random sampling methods for the retrospective analysis during January $1^{\text {st }}, 2001$ to December $31^{\text {st }}, 2004$, and a half of clinical MDRAB isolates monthly were selected, but no more than 10 isolates among each months, during the study period. Overall 440 MDRAB isolates were tested for the target gene intI1 during the study period.

\section{Pulse-field gel electrophoresis (PFGE) analysis}

The ampicillin-sulbactam resistance phenotype, IntI1 integrase positive genotype, and strains isolated within season of high infection rate were chosen as the selection criteria for listing candidates MDRAB strains. Standard protocol for PFGE analysis was employed for the A. baumannii isolates. Briefly A. baumannii were plated on blood agar and incubated in a $5 \% \mathrm{CO}_{2}$ atmosphere at $35^{\circ} \mathrm{C}$ for $16-24 \mathrm{~h}$. Plug slices were digested with $20 \mathrm{U}$ of SgrAI. The DNA fragments were then separated in $1 \%$ Seakem Gold agarose gels (FMC BioProducts) at $14^{\circ} \mathrm{C}$ using a Bio-Rad CHEF DRIII PFGE system (Bio-Rad Laboratories, Hercules, CA, USA). Gels were run in $0.5 \times$ Tris-borate-EDTA (TBE; $\mathrm{pH} 8)$ at a $120^{\circ}$ fixed angle and a fixed voltage $(6 \mathrm{~V} / \mathrm{cm})$, with pulse intervals from 4-40 s for $20 \mathrm{~h}$. Following staining and imaging, the chromosomal DNA restriction patterns produced by PFGE were interpreted using Tenover's categorization [16].

\section{Statistical analysis}

We used the finite mixture model on categorical dataset of antibiotic resistance phenotypes. The results were considered significant when the $P$ value was less than 0.05 . All data were analyzed using a SPSS software v10.0. We used the $X^{2}$ test on categorical data to analyze relations. We used the Spearman's correlation to analyze the relations between different parameters.

\section{Results}

During the past fifteen years, the overall hospital infection rate at $\mathrm{CCH}$ was $5-6 \%$, and the infection rate in the ICU fluctuated. During this time period, patients with $A B$ infections increased gradually at $\mathrm{CCH}$, especially in the ICU (data not shown). Between January $1^{\text {st }}, 2001$ and December $31^{\text {st }}, 2004$, two periods occurred during which the number of AB-infected patients increased. The Infection Control Office of $\mathrm{CCH}$ began to investigate the infection rate in the hospital and the ICU (Additional file 2). Several periods occurred during which the infection rate in the ICU increased, including June 2001, November 2001, February 2002, July 2002, September 2002, July 2003, November 2003, and March 2004. However, the reason for the gradual increase in $A B$ infection is still unknown, despite investigation. No significant changes in the numbers of MDRAB isolates and the carrying rate of IntI1 gene were observed when the data from 2001-2002 was compared to that of 2003-2004. 
Using a retrospective analysis and Koeleman's method, the results of amplifying the IntII gene from clinical 440 MDRAB isolates are shown in Table 1 . No positive correlation was observed between clinical MDRAB isolates and the presence of the IntI1 in the bacteria $(r=0.168$, $P=0.254$ ). Additionally, no positive correlation was observed between the infection rate in the ICU and the presence of IntI1 ( $\mathrm{r}=-0.107, P=0.468)$. Furthermore, no positive correlation was observed between the rate of hospital infection and the presence of integron $(r=-0.189$, $P=0.199)$. Thus, the presence of IntI1 integrase gene does not correlate with MDRAB prevalence and spread during the study period.

To evaluate the epidemiological features of the MDRAB isolates, selection criteria such as ampicillin-sulbactam resistance phenotype, IntI1 integrase positive genotype, and strains isolated within season of high infection rate were used for choosing candidates MDRAB strains (Table 2) Eight of the clinical MDRAB isolates (two isolates each year), which carried IntI1 positive genotype and ampicillinsulbactam resistance phenotypes were chosen in each July during the four study years. And, the other four clinical MDRAB isolates (one isolate each year), which is resistant to ampicillin-sulbactam but without carrying IntI1 gene, were also employed as reference strains during the same study period. The interpretation method for PFGE results is according to Tenover's criteria [16], and the statistic method is descriptive method (Table 2). Though PFGE results identified two predominant clones types (clone A, B) during the study year (Table 2, Additional file 3 ), the relationships between the predominant clones and the presence of the IntI1 did not have a significant association.

\begin{tabular}{|c|c|c|c|c|}
\hline Year & $\begin{array}{l}\text { Carrying-Int I1 } \\
\text { rate }\end{array}$ & $\begin{array}{l}\text { MDRAB } \\
\text { isolates } \\
\text { number }\end{array}$ & $\begin{array}{c}\text { ICU } \\
\text { infection rate }\end{array}$ & $\begin{array}{c}\text { Hospital } \\
\text { infection rate }\end{array}$ \\
\hline \multirow[t]{2}{*}{2001} & $r$ & 0.080 & -0.235 & -0.586 \\
\hline & $p$-value & 0.805 & 0.462 & 0.045 \\
\hline \multirow[t]{2}{*}{2002} & $r$ & 0.055 & -0.088 & -0.228 \\
\hline & $p$-value & 0.864 & 0.785 & 0.476 \\
\hline \multirow[t]{2}{*}{2003} & $r$ & 0.594 & 0.041 & 0.305 \\
\hline & $p$-value & 0.042 & 0.899 & 0.334 \\
\hline \multirow[t]{2}{*}{2004} & $r$ & -0.086 & -0.172 & -0.135 \\
\hline & $\mathrm{p}$-value & 0.790 & 0.594 & 0.675 \\
\hline \multirow[t]{2}{*}{ Overall } & $r$ & 0.168 & -0.107 & -0.189 \\
\hline & $p$-value & 0.254 & 0.468 & 0.199 \\
\hline
\end{tabular}

Notes: $r$ : Spearman's Correlation Coefficient, MDRAB: Multi-drug resistant A. baumannii.

\section{Discussion}

This study is the first epidemiological study to evaluate the involvement of class 1 integron for the appearance and spread of MDRAB in central Taiwan. MDRAB has been reported in Taiwan since the early $21^{\text {st }}$ century [17], and we also disclosed the same trend of MDRAB at the $\mathrm{CCH}$. After evidence-based literature review (Additional file 1), 2001-2002 was identified as the critical time period for the appearance and spread of MDRAB, so we engaged this study. Because of the structural diversity of RIs in AB, detecting these huge mobile elements is not simple. The epidemic class 1 integron-carrying MDRAB clone was reported in Taiwan [10]. Detection of this class 1 integron, which is located in an RI of $A B$, is convenient. To reveal the mechanism and to trace the rapid dissemination during the emergence of MDRAB, we choose the IntI1 integrase gene as the target for detection. However, the presence of IntI1 does not correlate with the emergence and spread of MDRAB in this study. The IntI1 gene is present in $81.3 \%$ of clinical $A B$ isolates at our institute [9] and $66.7 \%$ at Zhong's study [18].

In this study, we reported that the presence of IntI1 does not correlate with the emergence and spread of MDRAB, and it's different from previous study [19]. The reasons for no correlation between the intl1 gene and MDRAB could be multiple factors in this study. Firstly, since the structural complexity of RIs, the IntI1 gene may be not a good marker to trace the appearance and spread of MDRAB prior to the dissemination of class 1 integron-carrying MDRAB clones. Secondly, IntI1 is too common to be an accurate biological marker. The contributions of class 1 integrons in MDRAB involve their carried antibiotic cassettes. So, the presence of IntI1 integrase gene along does not correlate with the emergence and spread of MDRAB in this study. Further examinations were needed to reveal the correlation between their antibiotic cassettes and emergence of MDRAB.

The susceptibility rates of sulbactam-containing combinations (such as ampicillin-sulbactam) for AB were 90.492.7\% in Brauers's study [20], but the resistance rate of sulbactam-containing combinations (such as ampicillinsulbactam) for AB was 57.4-64.1\% in Taiwan Surveillance of Antimicrobial Resistance program [21]. The resistant rates to piperacillin/tazobactam and third geenration cephalosporines and fluoroquinolones for MDRAB strains in Taiwan were around $75 \%$, while the resistant rates to ampicillin-sulbactam for MDRAB strains in Taiwan was only $50 \%$ [21]. Since sulbactam resistance phenotpye of $\mathrm{AB}$ was shown to be unique in Taiwanese isolates, the resistance phenotpye of ampicillin-sulbactam was thus chosen as selection criteria for PFGE analysis candidates. With regard to genotyping for $\mathrm{AB}$, PFGE, multilocus sequence typing, and rep-PCR have been used in previous studies [22-24], while PFGE is still considered as the 
Table 2 Summary for results of PFGE fingerprint patterns and antibiotic susceptibility among clinical Acinetobacter baumannii isolates in the PFGE study

\begin{tabular}{|c|c|c|c|c|c|c|c|c|c|c|c|c|}
\hline Number & $\mathrm{P} 1^{1}$ & P2 & P3 & P4 & P5 & P6 & P7 & P8 & P9 & P10 & P11 & P12 \\
\hline Isolates year & 2001 & 2001 & 2002 & 2002 & 2003 & 2003 & 2004 & 2004 & 2001 & 2002 & 2003 & 2004 \\
\hline PFGE type & $A$ & $B$ & C & $B$ & A & $A$ & $B$ & $\mathrm{D}$ & $A$ & A & $E$ & A \\
\hline Carrying-Int 11 & + & + & + & + & + & + & + & + & - & - & - & - \\
\hline AN & $\mathrm{R}^{2}$ & $\mathrm{R}$ & $\mathrm{R}$ & $\mathrm{R}$ & $\mathrm{R}$ & $\mathrm{S}$ & $\mathrm{R}$ & $\mathrm{R}$ & $\mathrm{R}$ & $S$ & $\mathrm{R}$ & $S$ \\
\hline SAM & $\mathrm{R}$ & $\mathrm{R}$ & $\mathrm{R}$ & $\mathrm{R}$ & $\mathrm{R}$ & $\mathrm{R}$ & $\mathrm{R}$ & $\mathrm{R}$ & $\mathrm{R}$ & $\mathrm{R}$ & $\mathrm{R}$ & $\mathrm{R}$ \\
\hline CTZ & $S$ & $S$ & $\mathrm{R}$ & $\mathrm{S}$ & $\mathrm{R}$ & $\mathrm{S}$ & $\mathrm{R}$ & $\mathrm{S}$ & $\mathrm{R}$ & $S$ & $S$ & $\mathrm{R}$ \\
\hline LVF & $S$ & $S$ & $\mathrm{~S}$ & $S$ & $S$ & $\mathrm{~S}$ & $S$ & $\mathrm{~S}$ & $\mathrm{~S}$ & $S$ & $\mathrm{~S}$ & S \\
\hline IMP & $S$ & $\mathrm{~S}$ & $S$ & $\mathrm{~S}$ & $S$ & $S$ & $\mathrm{R}$ & $S$ & $\mathrm{~S}$ & $S$ & $\mathrm{R}$ & S \\
\hline PIP-TAZ & $\mathrm{S}$ & $\mathrm{R}$ & $\mathrm{S}$ & $\mathrm{R}$ & $\mathrm{S}$ & $\mathrm{R}$ & $\mathrm{S}$ & $\mathrm{R}$ & $\mathrm{S}$ & $\mathrm{R}$ & $S$ & $S$ \\
\hline CRO & $S$ & S & $\mathrm{R}$ & $S$ & $\mathrm{R}$ & $\mathrm{S}$ & $\mathrm{R}$ & $\mathrm{S}$ & $\mathrm{R}$ & $S$ & S & $\mathrm{R}$ \\
\hline
\end{tabular}

Notes:

${ }^{1} \mathrm{P}$ number is the Lane number of PFGE study.

${ }^{2}$ S: Susceptible; R: Resistant; I: Belonged to resistant.

${ }^{3}$ The susceptibility tests were performed using the Vitek-2 GN card (Biomerieux, Marcy l'Etoile, France). The results were interpreted using the CLSI breakpoints (Clinical and Laboratory Standards Institute Performance Standards for Antimicrobial Susceptibility testing).

${ }^{4}$ Abbreviation AN: amikacin; SAM: Ampicillin-sulbactam; CTZ: Ceftazidime; LVF: Levofloxacin; IMP: Imipenem-cilastatin; PIP-TAZ: Piperacillin-tazobactam; CRO: Ceftriaxone; colistin and tigecycline and polymyxin B were not provided by Vitek-2 System.

common standard for typing the outbreak-related isolates of AB [19]. In this study, PFGE results identified two predominant clones (clone A, B) (Figure 1) during the epidemic season. The result supports that PFGE is more accurate than IntI1-PCR to differentiate MDRAB isolates in central Taiwan. In this study, the reasons to choose three kinds of selection marker are as following: The first one is ampicillin/sulbactam resistant phenotype. Ampicillin/ sulbactam has been proven to be more efficacious than polymyxins in treating carbapenem-resistant $A$. baumannii infection [25]. Although resistance to ampicillin/sulbactam in $A$. baumannii has been reported in many countries [1] and authors disclosed one unique phenomenon is that some isolates of MDRAB, which were resistant to many broad-spectrum antibiotics (including ciprofloxacin, piperacillin-tazobactam, ceftazidime, cefepime, imipenem- cilastatin, amikacin) but susceptible to ampicillin/sulbactam between 2001 and 2002 [9]. No resistance mechanism was described in those studies. The second marker is intI1 integrase gene in class 1 integrons, and that is the target gene in this study. The last marker is timeline with recrudescent tendency. Because of a lot of clinical A. baumannii isolates, we needed to select the important isolates to study. According to the Additional file 2 (which shows the infection rate of the hospital and the ICU during 2001-2004), the strains isolated within season of high infection rate are from summer season during the four study period. It would be interesting to know how many of the nosocomial or healthcare-associated MDRAB isolates belongs to the same fingerprint type, and we should be cautious to those predominant clones, which spreading among hospitals and disseminating their integrons involved

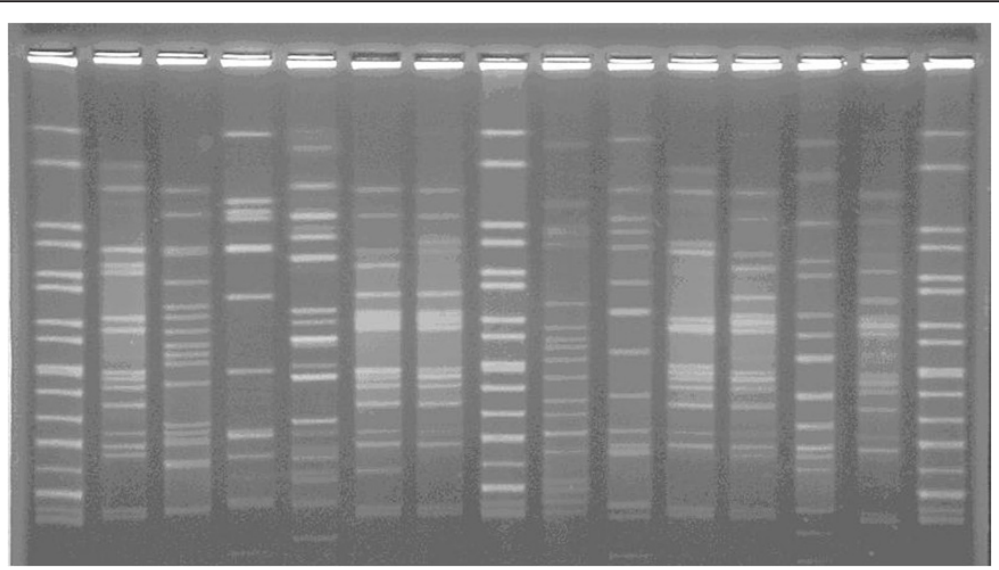

Figure 1 PFGE fingerprints of 12 multi-resistant A. baumannii (MDRAB) isolates with resistance to ampicillin-sulbactam. 
gene cassettes. Further examinations are needed to reveal the facts.

Concerning infection rate of the hospital and the ICU, it was also examined in this study (Table 1, Additional file 2). Several periods occurred during which the infection rate in the ICU increased, including June 2001, November 2001, February 2002, July 2002, September 2002, July 2003, November 2003, and March 2004. New staff has begun to work after orientation and training in June and September since 1990 at our institute. Additionally, budget cuts resulted in downsizing and changes in staffing, and many buildings were erected at our institute. Thus, further study is needed to elucidate the reasons and factors for the increase in clinical MDRAB isolates at central Taiwan at the period of the early $21^{\text {st }}$ century.

\section{Conclusion}

In this study, the emergence of MDRAB in a Taiwanese hospital was traced by the correlated between detection of IntI1 and existence of MDRAB. This is the first crosssectional, clinical epidemiological study on the involvement of class 1 integron in the emergence and spread of MDRAB in Central Taiwan. Though the existence of intI1 gene is not suitable for tracing MDRAB emergence that occurred at $\mathrm{CCH}$, two predominant clones were identified from PFGE study, and subsequent studies to determine whether these clones are responsible for the original nosocomial infection are needed.

\section{Additional files}

Additional file 1: The literature summary of clinical multidrugresistant Acinetobacter baumannii isolates between 2002 and 2004 in Taiwan.

Additional file 2: Infection rate of the hospital and the ICU during 2001-2004.

Additional file 3: PFGE fingerprints of 12 multi-resistant $A$. baumannii (MDRAB) isolates with resistance to ampicillin-sulbactam.

\section{Abbreviations}

AB: Acinetobacter baumannii; CC: Changhua County; CCH: Changhua Christian Hospital; HGT: Horizontal gene transfer; MDRAB: Multidrug-resistant Acinetobacter baumannii; PFGE: Pulsed-field gel electrophoresis; Rls: Resistant islands.

\section{Competing interests}

The authors declare that they have no competing interests.

\section{Authors' contributions}

$\mathrm{CHC}$ and $\mathrm{CCH}$ designed and performed this study. $\mathrm{CHC}$ served the patients. $\mathrm{CHC}$ and $\mathrm{CCH}$ wrote the manuscript. Both authors read and approved the final manuscript.

\section{Acknowledgements}

The authors thank Changhua Christian Hospital for the kind gift of the clinical Acinetobacter baumannii isolates. We thank Choiu CS and Laiu JC of the Central Branch Office, Center for Disease Control, Taichung. We also thank Lin LC, Huang CW, and Chu WC for technical assistance. This study was partially supported by a grant from the Changhua Christian Hospital. Authors thank Liu CE, Lin KH for the assistance of clinical service for those patients. The authors thank Li-Chen Lin for technical assistance, and thank Yu-Jun Chang for statistical assistance. Both authors thank the assistant of the Clinical Microbiology Laboratory of Changhua Christian Hospital. This research project would not have been possible without the support of many people. Both authors wish to express their gratitude to staffs of Division of Infectious Diseases and Division of Critical Care Medicine of Changhua Christian Hospital who were abundantly helpful and offered patient care, invaluable assistance, and support.

\section{Author details}

'Division of Infectious Diseases, Department of Internal Medicine, Changhua Christian Hospital, Changhua 500, Taiwan. ${ }^{2}$ Infection Control Committee, Department of Internal Medicine, Changhua Christian Hospital, Changhua 500, Taiwan. ${ }^{3}$ Department of Nursing, College of Medicine \& Nursing, Hung Kuang University, No. 1018,Sec. 6, Taiwan Boulevard, Shalu District, Taichung 43302, Taiwan. ${ }^{4}$ College of Life Science, National Chung Hsing University, 250 Kuo-Kuang Road, Taichung 402, Taiwan.

Received: 29 April 2014 Accepted: 8 August 2014

Published: 14 August 2014

\section{References}

1. Perez F, Hujer AM, Hujer KM, Decker BK, Rather PN, Bonomo RA: Global challenge of multidrug-resistant Acinetobacter baumannii. Antimicrob Agents Chemother 2007, 51:3471-3484

2. Peleg AY, Seifert $H$, Paterson DL: Acinetobacter baumannii: emergence of a successful pathogen. Clin Microbiol Rev 2008, 21:538-582.

3. Valenzuela JK, Thomas $L$, Partridge $S R$, van der Reijden T, Dijkshoorn $L$, Iredell J: Horizontal gene transfer in a polyclonal outbreak of carbapenem-resistant Acinetobacter baumannii. J Clin Microbio/ 2007, 45:453-460.

4. Dobrindt U, Hochhut B, Hentschel U: Genomic islands in pathogenic and environmental microorganisms. Nat Rev Microbiol 2004, 2:414-424.

5. Adams MD, Chan ER, Molyneaux ND: Genomewide analysis of divergence of antibiotic resistance determinants in closely related isolates of Acinetobacter baumannii. Antimicrob Agents Chemother 2010, 54:3569-3577.

6. Chen CC, Lin YC, Sheng WH: Genome sequence of a dominant, multidrug-resistant Acinetobacter baumannii strain, TCDC-AB0715. J Bacteriol 2011, 193:2361-2362.

7. Fournier $P E$, Vallenet $D$, Barbe $V$ : Comparative genomics of multidrug resistance in Acinetobacter baumannii. PLoS Genet 2006, 2:e7.

8. Zhu L, Yan Z, Zhang Z, Zhou Q, Zhou J, Wakeland EK, Fang X, Xuan Z, Shen D, Li QZ: Complete genome analysis of three acinetobacter baumannii clinical isolates in China for insight into the diversification of drug resistance elements. PLoS One 2013, 8:e66584.

9. Chen $\mathrm{CH}$, Young TG, Huang CC: Predictive biomarkers for drug-resistant Acinetobacter baumannii isolates with bla(TEM-1), AmpC-type bla and integrase 1 genotypes. J Microbiol Immunol Infect 2006, 39:372-379.

10. Huang LY, Chen TL, Lu PL, Tsai CA, Cho WL, Chang FY, Fung CP, Siu LK: Dissemination of multidrug-resistant, class 1 integron-carrying Acinetobacter baumannii isolates in Taiwan. Clin Microbiol Infect 2008, 14:1010-1019.

11. Koeleman JGM, Stoof J, van der Bijl M, Vandenbroucke-Grauls CMJE, Savelkoul PHM: Identification of epidemic strain of Acinetobacter baumannii by integrase gene PCR. J Clin Microbiol 2001, 39:8-13.

12. Severino P, Magalhaes VD: Integrons as tools for epidemiological studies. Clin Microbiol Infect 2004, 10:156-162.

13. Khuri-Bulos NA, Shennak M, Agabi S, Saleh S, Al Rawashdeh S, Al Ghanem S, Al Adham M, Faori l, Abu Khader I: Nosocomial infections in the intensive care units at a university hospital in a developing country: comparison with national nosocomial infections surveillance intensive care unit rates. Am J Infect Control 1999, 27:547-552.

14. Clinical and Laboratory Standards Institute: Performance Standards for Antimicrobial Susceptibility Testing, 15th Informational Supplement. M100-S15. Wayne, PA: CLSI; 2005.

15. APIC: "Guide to the elimination of multidrug -resistant acinetobacter baumannii transmission in healthcare settings". 2010, http://www.apic. org/EliminationGuides.

16. Tenover FC: Plasmid fingerprinting. a tool for bacterial strain identification and surveillance of nosocomial and community-acquired infections. Clin Lab Med 1985, 5:413-436. 
17. Hsueh PR, Teng L, Chen CY, Chen WH, Yu CJ, Ho SW, Luh KT: Pandrugresistant Acinetobacter baumannii causing nosocomial infections in a university hospital, Taiwan. Emerg Infect Dis 2002, 8:827-832.

18. Zhong $\mathrm{Q}, \mathrm{Xu} \mathrm{W}, \mathrm{Wu} \mathrm{Y}, \mathrm{Xu} \mathrm{H}$ : Clonal spread of carbapenem non-susceptible acinetobacter baumannii in an intensive care unit in a Teaching Hospital in China. Ann Lab Med 2012, 32:413-419. 25:719-21.

19. Lin MF, Liou ML, Tu CC, Yeh HW, Lan CY: Molecular epidemiology of integron-associated antimicrobial gene cassettes in the clinical isolates of Acinetobacter baumannii from northern Taiwan. Ann Lab Med 2013, 33:242-247.

20. Brauers J, Frank U, Kresken M, Rodloff AC, Seifert H: Activities of various beta-lactams and beta-lactam/beta-lactamase inhibitor combinations against Acinetobacter baumannii and Acinetobacter DNA group 3 strains. Clin Microbiol Infect 2005, 11:24-30.

21. Kuo SC, Chang SC, Wang HY, Lai JF, Chen PC, Shiau YR, Huang IW, Lauderdale TL: TSAR Hospitals. emergence of extensively drug-resistant Acinetobacter baumannii complex over 10 years: nationwide data from the Taiwan Surveillance of Antimicrobial Resistance (TSAR) program. BMC Infect Dis 2012, 12:200.

22. Seifert H, Dolzani L, Bressan R, van der Reijden T, van Strijen B, Stefanik D, Heersma H, Dijkshoorn L: Standardization and interlaboratory reproducibility assessment of pulsed-field gel electrophoresis-generated fingerprints of Acinetobacter baumannii. J Clin Microbiol 2005, 43:4328-4335.

23. Bartual SG, Seifert H, Hippler C, Luzon MA, Wisplinghoff H, Rodríguez-Valera F: Development of a multilocus sequence typing scheme for characterization of clinical isolates of Acinetobacter baumannii. J Clin Microbiol 2005, 43:4382-4390.

24. Higgins PG, Hujer AM, Hujer KM, Bonomo RA, Seifert H: Interlaboratory reproducibility of DiversiLab rep-PCR typing and clustering of Acinetobacter baumannii isolates. J Med Microbio/ 2012, 61:137-141.

25. Betrosian AP, Frantzeskaki F, Xanthaki A, Douzinas EE: Efficacy and safety of high-dose ampicillin/sulbactam vs. colistin as monotherapy for the treatment of multidrug resistant Acinetobacter baumannii ventilatorassociated pneumonia. J Infect 2008, 56:432-436.

doi:10.1186/1477-5751-13-15

Cite this article as: Chen and Huang: Tracing the emergence of multidrug-resistant Acinetobacter baumannii in a Taiwanese hospital by evaluating the presence of integron gene intl1. Journal of Negative Results in BioMedicine 2014 13:15.

\section{Submit your next manuscript to BioMed Central and take full advantage of:}

- Convenient online submission

- Thorough peer review

- No space constraints or color figure charges

- Immediate publication on acceptance

- Inclusion in PubMed, CAS, Scopus and Google Scholar

- Research which is freely available for redistribution

Submit your manuscript at www.biomedcentral.com/submit
( Biomed Central 